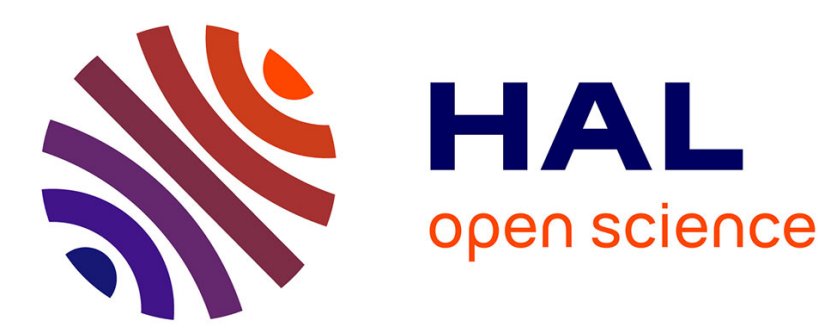

\title{
Formation à l'étranger, fuite des cerveaux et bien-être national: Enjeux et paradoxes dans une perspective microéconomique
}

Bernard Franck, Robert F. Owen

\section{To cite this version:}

Bernard Franck, Robert F. Owen. Formation à l'étranger, fuite des cerveaux et bien-être national : Enjeux et paradoxes dans une perspective microéconomique. 2010. hal-00472472

HAL Id: hal-00472472

https://hal.science/hal-00472472

Preprint submitted on 12 Apr 2010

HAL is a multi-disciplinary open access archive for the deposit and dissemination of scientific research documents, whether they are published or not. The documents may come from teaching and research institutions in France or abroad, or from public or private research centers.
L'archive ouverte pluridisciplinaire HAL, est destinée au dépôt et à la diffusion de documents scientifiques de niveau recherche, publiés ou non, émanant des établissements d'enseignement et de recherche français ou étrangers, des laboratoires publics ou privés. 


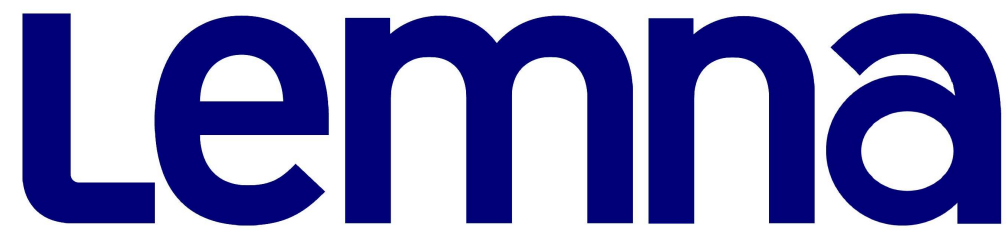

EA 4272

\title{
Formation à l'étranger, fuite des cerveaux et bien-être national : Enjeux et paradoxes dans une perspective microéconomique
}

\author{
Bernard Franck $\left(^{*}\right)$ \\ Robert Owen $\left.{ }^{* *}\right)$
}

2010/18

(*)Faculté Sciences Economiques et Gestion - Université de Caen-Basse Normandie - CREM

$\left(^{* *}\right)$ LEMNA - Université de Nantes

Laboratoire d'Economie et de Management Nantes-Atlantique Université de Nantes

Chemin de la Censive du Tertre - BP 52231

44322 Nantes cedex 3 - France

www.univ-nantes.fr/iemn-iae/recherche 


\title{
Formation à l'étranger, fuite des cerveaux et bien-être national:
}

\section{Enjeux et paradoxes dans une perspective microéconomique}

\author{
par \\ Bernard Franck* \\ et \\ Robert F. Owen** \\ Mars 2010
}

\begin{abstract}
*Faculté des Sciences Economiques et de Gestion, Université de Caen-Basse Normandie et CREM (UMR CNRS 6211), Esplanade de la Paix, 14032 Caen Cedex, France. Téléphone: 33-2-31-56-56-95, Fax: 33-2-31-93-61-94.

E-mail : bernard.franck@unicaen.fr
\end{abstract}

**LEMNA, Institut d'Economie et de Management de Nantes - I.A.E., Université de Nantes, Chemin de la Censive du Tertre, B. P. 52231, 44322 Nantes Cedex 3, Téléphone: 33-2-40-14-17-61 (direct) et 33-2-40-14-17-17. Fax : 33-1-39-21-90-96. Mobile: 33676878853

E-mail : $\underline{\text { robertfowen@gmail.com }}$ 
Formation à l'étranger, fuite des cerveaux et bien-être national: Enjeux et paradoxes dans une perspective microéconomique

\title{
Résumé
}

Pour étudier les migrations internationales de travailleurs qualifiés, nous construisons un modèle à deux pays : pays d'origine et pays d'accueil. Un étudiant du pays d'origine peut soit entreprendre des études locales, soit aller étudier à l'étranger où il a par la suite une certaine probabilité de trouver un travail qualifié. Son choix dépendra de ses capacités, de la qualité des systèmes éducatifs, du coût relatif des études et des conditions de fonctionnement du marché du travail dans les deux pays. La possibilité de migrer entraîne des changements dans le bien-être du pays d'origine que nous cherchons à évaluer. Nous envisageons également la possibilité que les autorités favorisent les études à l'étranger grâce à un système de bourses qui vont potentiellement accroître ou diminuer le bien-être national.

Mots-clefs : migrations internationales, fuite des cerveaux, formation du capital humain, coûts irréversibles, bourses d'étude

Code JEL : F22, F15

International Human Capital Formation, Brain Drain and National Welfare : Economic Stakes and Paradoxes in a Microeconomic Perspective

\begin{abstract}
$\underline{\text { Abstract }}$
A two-country, two-period model of international migration highlights microeconomic foundations for the evaluation of brain drain and brain gain effects. Ex ante choices regarding whether to study at home or abroad depend on the relative quality of university systems, individuals' abilities, sunk costs of education, as well as labour market conditions in both countries and an individual's anticipated employment prospects. Migration results in economic welfare changes for the home country, which are shown to be a complex function of parameters related to the cost of studies, the distribution of graduates' skills, relative employment conditions and the efficacy of government grant policies supporting foreign studies.
\end{abstract}

Key words: human capital formation, brain drain, brain gain, international migration, sunk costs, educational grants

JEL classification codes: F22, F15 


\section{$\underline{\text { Section I : Introduction }}$}

L'effet destructeur des migrations de personnel qualifié («brain drain ") sur la croissance et le bien-être du pays d'origine est bien connu depuis la contribution de Bhagwati et Hamada (1974), même si des études déjà anciennes ont reconnu que le phénomène pouvait présenter certains aspects positifs pour ce pays tels que les transferts financiers effectués par les migrants vers celui-ci, et le retour éventuel de travailleurs qui se sont formés sur le tas dans le pays d'accueil. Plus récemment, des travaux initiés par Mountford (1997) et Stark, Helmenstein et Prskawetz (1997) ont mis en évidence une autre source potentiellement importante d'avantages («brain gain »), indépendante des retours au pays. Ces travaux (baptisés par Schiff (2006) « la nouvelle littérature sur le brain drain ») montrent en effet que, bien que les migrations constituent une perte de talents pour l'économie locale, elles peuvent également engendrer un accroissement du niveau global de l'éducation dans le pays considéré, dans la mesure où la perspective de pouvoir trouver du travail à l'étranger incite les individus à un plus grand investissement en capital humain, sans que ceci ait nécessairement pour conséquence un départ effectif.

Certains de ces travaux sont d'ordre macroéconomique et raisonnent en termes d'agents représentatifs. Mais d'autres considèrent des agents individuels qui font des choix en matière de niveau optimal d'investissement en capital humain. Stark, Helmenstein et Prskawetz (1997) ont proposé un cadre d'analyse montrant comment, quand il est possible d'émigrer, les choix éducatifs vont déterminer le salaire d'un individu sur le marché du travail étranger. Dans d'autres modélisations, le migrant potentiel prend en compte une probabilité de trouver du travail à l'étranger qui est identique pour tous les individus (Stark, Helmenstein et Prskawetz, 1998) ou contrainte par un seuil minimal de qualification ( Stark, 2004). Mountford (1997) et Beine, Docquier et Rapoport (2001, 2008) proposent des modèles dans lesquels la décision de l'individu est de forme binaire : entreprendre ou non des études, alors que la probabilité de trouver un emploi à l'extérieur est exogène. Les migrants sont alors choisis de façon aléatoire. Au contraire, chez Chiswick (1999), les migrants s'auto-sélectionnent dans la mesure où, étant donné deux catégories d'individus qui ont des capacités différentes, le rendement de la migration est plus élevé pour les individus d'aptitude élevée que pour les plus médiocres.

L'évaluation des effets négatifs («brain drain ») et positifs («brain gain ») des migrations est fait dans la littérature en observant l'impact des migrations sur différents objectifs mais sans prendre en compte explicitement une fonction de bien-être. Les auteurs montrent notamment que les migrations influencent le taux de croissance de l'économie du pays d'origine (Beine, Docquier et Rapoport, 2001), le niveau moyen d'éducation 
( Stark et al. , 1997, 1998, Lien et Wang, 2005), la productivité moyenne (Mountford, 1997) ou encore les salaires des non-migrants (Stark, 2004).

Bien qu'il existe maintenant un nombre croissant d'études empiriques qui estiment différentes dimensions de l'impact potentiel du brain drain et du brain gain, on peut constater l'absence de consensus au sujet de l'importance des effets positifs des migrations sur le pays d'origine. En particulier, Beine, Docquier et Rapoport $(2001,2008)$ trouvent que la proportion de migrants doit être faible pour que de tels effets se manifestent. Selon Schiff (2006), les études préliminaires entreprises par la Banque Mondiale ne montrent aucun impact significativement positif, et un résultat similaire est obtenu par Groizard et Lull (2006).

Rosenzweig (2006) a critiqué les études existantes sur deux points importants qui apparaissent comme particulièrement pertinents pour justifier la modélisation proposée ici. Tout d'abord, il prétend que l'impact potentiel du risque d'émigration concernant des personnes qui ont suivi des études supérieures dans le pays d'origine est minimal. D'autre part, il observe que la littérature ignore le caractère endogène de la probabilité d'émigration en ajoutant que le choix de localisation de l'éducation affecte significativement la probabilité que la personne aille travailler à l'étranger ${ }^{1}$ . En continuité avec la modélisation proposée par Franck et Owen (2009), il nous semble également que la mobilité des travailleurs qualifiés peut être affectée par le choix préalable entre études locales et études à l'étranger.

Nous construisons à cet effet un modèle à deux pays, dans lequel les étudiants du pays d'origine ont le choix entre poursuivre des études supérieures localement, ou dans le pays d'accueil ( dont le système éducatif est supposé de meilleure qualité). Dans ce dernier cas, ils ont, une fois diplômés, la possibilité de se présenter sur le marché du travail étranger, alors que des études purement locales, compte tenu de leur qualité et d'autres facteurs (comme, par exemple, l'influence des réseaux professionnels) ne permettent pas cet accès. Les éléments distinctifs du modèle proposé sont principalement les suivants :

1) Chaque individu du pays d'origine doit choisir l'endroit où il va entreprendre des études supérieures : sur place ou à l'étranger. Les études à l'étranger lui conféreront, à capacité donnée, et une fois embauché, une plus grande productivité que s'il avait suivi des études locales. En supposant qu'il arrive à trouver un emploi dans le pays d'accueil, il $\mathbf{y}$ restera, car les salaires y sont plus élevés que dans le pays

\footnotetext{
${ }^{1}$ Bien que le cadre d'analyse proposé par Rosenzweig n'introduise pas des différences dans les capacités individuelles, ses résultats empiriques sont cohérents avec un certain nombre des hypothèses de formalisation qui seront explicitées par la suite. Il mentionne notamment que l'espoir de trouver du travail dans le pays hôte constitue une motivation pour aller étudier à l'étranger, et que les différences de qualité entre les systèmes universitaires sont aussi un facteur de la décision .
} 
d'origine, participant ainsi à la fuite des cerveaux. En revanche, au cas où il ne trouverait pas de travail à l'étranger, il rentrera et bénéficiera tout de même d'un différentiel de productivité et de salaire par rapport aux diplômés locaux, ce qui constitue l'essence du «brain gain ». Il faut donc remarquer que la notion de «brain gain " qui apparaît dans notre modèle est distincte de celle qui est utilisée habituellement dans la littérature puisque les gains de productivité sont liés ici non pas à l'éducation locale mais à l'éducation à l'étranger.

2) La probabilité d'être embauché à l'étranger représente un élément important dans la modélisation du choix individuel de localisation des études. Contrairement aux autres modèles dans lesquels cette probabilité est exogène et identique pour tous les diplômés, elle est ici supposée dépendre de la qualification de l'individu représentée par sa productivité future, dépendant elle-même de ses capacités. Ainsi les migrants sont positivement autoselectionnés comme chez Chiswick (1999).

3) Le critère choisi pour évaluer les effets du couple «brain drain / brain gain» est l'impact sur le bien-être du pays d'origine. Celui-ci est matérialisé par la valeur ajoutée additionnelle engendrée dans le pays par la possibilité d' étudier à l'étranger compte tenu de l'accroissement de productivité des diplômés revenant dans le pays et aussi du coût social supplémentaire causé par ces études. On néglige ici les transferts de salaire effectués par les émigrés vers leur pays d'origine ${ }^{2}$.

4) Dans la mesure où les études à l'étranger développent la productivité et peuvent ainsi avoir une incidence positive sur le bien-être, les autorités publiques du pays d'origine peuvent souhaiter les encourager en subventionnant les étudiants qui souhaitent partir mais en sont dissuadés par le coût trop élevé de ces études par rapport à leur rendement anticipé. En supposant que les autorités ont une information parfaite sur les capacités des étudiants, on constate que sous certaines conditions, l'attribution de bourses exerce effectivement une influence positive sur l'avantage collectif, mais une question se pose alors : faut-il subventionner les étudiants les plus brillants? On montrera que cela n'est pas toujours le cas.

\footnotetext{
${ }^{2}$ Si ces transferts sont positifs, on sous-estime ainsi les effets favorables au bien-être. Cependant, en période de crise, ils peuvent être relativement moins importants ou même négatifs, comme on l'a observé récemment.
} 
La suite de l'article est structurée de la façon suivante : Nous présentons dans la section II la modélisation qui aboutit à l'expression de la variation de bien-être engendrée par la possibilité d'accomplir des études à l'étranger ainsi qu'un certain nombre d'exercices de statique comparative pour analyser l'influence des paramètres du modèle sur cette variation de bien-être. On envisage dans la section III l'instauration d'un système de bourses attribuées par le pays d'origine à certains de ses étudiants désireux de partir à l'étranger: l'administration étant supposée avoir une information parfaite sur les capacités des étudiants, on caractérise les candidats qui devront être sélectionnés de façon à obtenir l'accroissement maximum de bien-être.

\section{Section II: Le modèle de base}

\section{1. Les comportements individuels et les flux migratoires}

On décrit ici le comportement d'un agent individuel qui doit décider s' il va entreprendre des études à l'étranger en fonction de ses perspectives futures d'emploi.

On suppose que si l'individu choisit de poursuivre ses études dans son pays d'origine, il n'aura pas accès au marché du travail du pays étranger. En revanche, s'il étudie dans le pays étranger, il acquière une qualification supérieure qui lui ouvre la perspective de trouver du travail dans ce pays grâce à sa plus grande productivité et à d'autres considérations qui permettent un accès plus facile au marché du travail à l'étranger (politiques de visas et effets des réseaux professionnels).

Plus précisément, dans une population totale de $\mathrm{N}$ individus, $\mathrm{N}_{0}$ représente le nombre d'individus qui ne partent pas faire d'études à l'étranger, et qui donc seront absorbés par le marché du travail local. Le reste de la population, $\mathrm{N}^{*}$, étudie à l'étranger. Parmi ceux-ci, $\mathrm{N}_{1}$ * représente le nombre d'individus qui vont trouver du travail et donc rester à l'étranger, $\mathrm{N}_{1}$ correspond à ceux qui vont rentrer travailler dans le pays d'origine. Ainsi, $\mathrm{N}_{1} *$ et $\mathrm{N}_{1}$ représentent respectivement les niveaux de la fuite et du retour des cerveaux.

Les individus qui constituent la population de base diffèrent en matière de capacités intellectuelles. Il existe par ailleurs une fonction de production éducative qui transforme ces capacités en qualifications représentées par les productivités individuelles. Le passage des capacités 
aux productivités est dépendant de la qualité des deux systèmes éducatifs. On suppose ici pour simplifier que, lorsqu'un individu poursuit des études locales, sa productivité future est $\mathrm{e}_{0}$ quelles que soient ses capacités. En ce qui concerne ceux qui étudient à l'étranger, leur productivité est répartie uniformément sur un segment $\left[\mathrm{e}_{1}, \mathrm{e}_{2}\right]$ avec $\mathrm{e}_{0}<\mathrm{e}_{1}$.

Ainsi, la décision d'entreprendre des études à l'étranger offre la perspective de gains de productivité plus élevés. Cependant elle est aussi plus coûteuse. Soit $I *$ le coût pour l'étudiant de l'éducation à l'étranger et $I_{0}$ le coût des études locales, $i=I^{*}-I_{0}$ est le coût supplémentaire lié au départ de l'étudiant.

Lorsqu'un individu envisage de partir étudier à l'étranger et donc d'assumer les coûts irréversibles correspondants, il doit bien entendu tenir compte de ses perspectives futures d'emploi et de rémunération. On suppose ici qu'une fois diplômé, il peut toucher, s'il obtient un emploi à l'étranger, un salaire $\mathrm{w}^{*}$ plus élevé que celui qu'on peut lui proposer dans le pays d'origine, $w_{1}$. Cependant il n'est pas assuré d'être embauché à l'étranger. L'individu $k$, appartenant à la population $N^{*}$ des étudiants éduqués à l'extérieur, n'a qu'une probabilité $p_{k}$ d'être recruté sur le marché du travail étranger. Cette probabilité joue un rôle fondamental dans le modèle pour déterminer l'étendue du «brain drain » et du « brain gain » comme on peut le constater en considérant les cas extrêmes : $\mathbf{S i} \mathbf{p}_{\mathbf{k}}=$ $\mathbf{0} \forall \mathbf{k}$, les individus n'ont aucune chance de trouver du travail à l'étranger ; ils vont donc tous rentrer au pays ${ }^{3}$ et le gain sera maximum. En revanche, si $\mathbf{p}_{\mathrm{k}}=1 \forall \mathrm{k}$, tous les diplômés trouvent du travail à l'étranger, donc le gain pour le pays est nul et la fuite des cerveaux est maximale.

En supposant toujours $p_{k}=p$ identique pour tous les individus mais maintenant comprise entre 0 et 1 , tous vont partir si $p$ est suffisamment élevée mais seule la fraction 1 - $p$ rentrera travailler dans le pays d'origine. Il y aura donc simultanément du brain drain et du brain gain dans les proportions p et 1-p.

En fait dans ce modèle on suppose que cette probabilité est différente selon les individus, et plus précisément qu'elle est liée de façon croissante à leur qualification mesurée par leur productivité. Les valeurs limites de celle-ci correspondant respectivement aux valeurs 0 et 1 de la probabilité sont $E_{1}$ et $\mathbf{E}_{2}$. Ces valeurs limites sont liées aux conditions régnant sur le marché du travail étranger. Par exemple des valeurs plus faibles de E2 pourraient correspondre à une situation dans laquelle il $y$ a un déficit relatif de travailleurs qualifiés. Des valeurs plus élevées (plus basses) des deux paramètres pourraient également être associées à des politiques d'immigration plus restrictives (plus laxistes) en fonction des qualifications des individus.

\footnotetext{
${ }^{3}$ On suppose que le marché du travail local peut absorber la totalité de ce personnel qualifié .
} 
La distribution des qualifications des individus en relation avec les exigences du marché du travail étranger est résumée dans la Figure 1 : 


\section{Figure 1}

$\underline{\text { Structure des qualifications }}$

\begin{tabular}{ccc|c|c}
\hline & & & & \\
$\mathbf{e}_{0}$ & $\mathbf{E}_{1}$ & $\mathbf{e}_{1}$ & $\mathbf{e}_{2}$ & $\mathbf{E}_{2}$
\end{tabular}

Ainsi on suppose $E_{1}<e_{1}<e_{2}<E_{2}$, ce qui veut dire que tout étudiant diplômé à l'étranger dispose d'une probabilité non nulle d'y trouver du travail. Si l'on donne une forme linéaire à la relation probabilité productivité, on peut écrire que pour tout individu $k$ on a :

(1.) $\mathrm{p}_{\mathrm{k}}=\mathrm{p}\left(\mathrm{e}_{\mathrm{k}}\right)=\frac{\left(\mathrm{e}_{\mathrm{k}}-\mathrm{E}_{1}\right)}{\left(\mathrm{E}_{2}-\mathrm{E}_{1}\right)}$

Rappelons également que pour simplifier on suppose $\mathrm{e}_{0}<\mathrm{E}_{1}$ : les individus qui n'ont fait que des études locales ne peuvent pas trouver du travail à l'étranger ${ }^{4}$.

A la suite de leurs études, les étudiants éduqués à l'étranger sont incités à y rester en raison des salaires ( $\left.w^{*}\right)$ qui y sont plus élevés que sur le marché du travail local $\left(w_{1}\right)$. Contrairement à la probabilité , ces salaires sont supposés exogènes, donc en particulier indépendants du niveau de qualification atteint c'est-à-dire de la productivité. De plus, $w_{1}$ est supposé supérieur à la fois à $\mathrm{w}_{0}$, salaire offert aux diplômés locaux et à $\mathrm{w}_{0} *$, salaire offert aux non-qualifiés à l'étranger ${ }^{5}$. Ceci assure que si un étudiant ne trouve pas de travail qualifié sur le marché étranger, il va rentrer chez lui. ${ }^{6}$. On néglige ainsi le scénario parfois appelé «brain waste» dans lequel un diplômé préfère occuper un emploi non qualifié à l'étranger plutôt que de rentrer ${ }^{7}$.

La Figure 2 résume ces hypothèses :

\footnotetext{
${ }^{4}$ Cela peut être du à des raisons concernant la spécificité des systèmes éducatifs locaux, la non-appartenance des étudiants étrangers à des réseaux facilitant l'insertion professionnelle ou des politiques restrictives de l'immigration favorisant les étudiants formés sur place.

${ }^{5}$ On suppose un taux de change fixe et en conséquence on peut considérer pour simplifier que les salaires dans les deux pays sont censés être exprimés dans la même unité monétaire.
}

\footnotetext{
${ }^{6}$ Naturellement, des facteurs non-économiques (comme le mariage et d'autres considérations familiales, ainsi que les affinités culturelles etc.) peuvent également influer sur cette décision, mais ils peuvent jouer dans les deux sens selon les circonstances.

${ }^{7}$ Fan et Stark (2007) proposent une analyse du chômage des diplômés, mais en s'intéressant seulement au pays d'origine, supposé en développement.
} 
Figure 2

$\underline{\text { Structure des salaires en fonction de la localisation et de la }}$ qualification

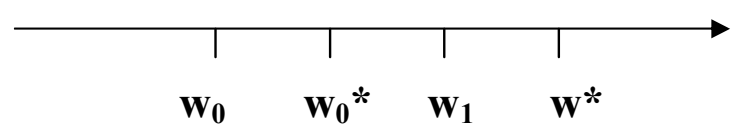

Le choix de partir ou non à l'étranger implique, pour un individu $k$, un arbitrage entre le coût plus élevé des études à l'étranger et une espérance de gains futurs supérieure, celle-ci tenant compte de la probabilité $p_{k}$ de trouver du travail à l'étranger. Il choisira de partir si

(2.) $\mathrm{p}_{\mathrm{k}} \mathrm{W}^{*}+\left(1-\mathrm{p}_{\mathrm{k}}\right) \mathrm{W}_{1}-\mathrm{I}^{*}>\mathrm{W}_{0}-\mathrm{I}_{0}$

Cela revient à dire que l'individu $k$ décidera d'aller étudier à l'étranger si sa probabilité individuelle d'y trouver du travail, $p_{k}$ est supérieure à une certaine limite, $\bar{p}$, donnée par

$$
\begin{aligned}
& \text { (3.) } \overline{\mathrm{p}}=\frac{\mathrm{i}-\left(\mathrm{w}_{1}-\mathrm{w}_{0}\right)}{\mathrm{w}^{*}-\mathrm{W}_{1}} \text { si } \frac{\mathrm{i}-\left(\mathrm{w}_{1}-\mathrm{w}_{0}\right)}{\mathrm{w}^{*}-\mathrm{w}_{1}} \in[0,1] \\
& \overline{\mathrm{p}}=0 \quad \text { si } \frac{\mathrm{i}-\left(\mathrm{w}_{1}-\mathrm{w}_{0}\right)}{\mathrm{w}^{*}-\mathrm{w}_{1}}<0 \text {, soit } \mathrm{i}<\mathrm{w}_{1}-\mathrm{w}_{0} \\
& \overline{\mathrm{p}}=1 \quad \text { si } \frac{\mathrm{i}-\left(\mathrm{w}_{1}-\mathrm{w}_{0}\right)}{\mathrm{w}^{*}-\mathrm{w}_{1}}>1 \text {, soit } \mathrm{i}>\mathrm{w}^{*}-\mathrm{w}_{0}
\end{aligned}
$$

D'après l'équation (1), à cette probabilité limite est associé un seuil de productivité $\widetilde{\mathrm{e}}=\left(\mathrm{E}_{2}-\mathrm{E}_{1}\right) \overline{\mathrm{p}}+\mathrm{E}_{1}$. Cependant, $\widetilde{\mathrm{e}}$ ' appartient pas nécessairement à l'intervalle des niveaux de productivité susceptibles d'être atteints à la suite des études, $\left[\mathrm{e}_{1}, \mathrm{e}_{2}\right]$, de telle sorte que, en toute rigueur, le seuil effectif de productivité est $\overline{\mathrm{e}}$ tel que

$$
\begin{aligned}
& \text { (4.) } \overline{\mathrm{e}}=\widetilde{\mathrm{e}}=\left(\mathrm{E}_{2}-\mathrm{E}_{1}\right) \overline{\mathrm{p}}+\mathrm{E}_{1} \quad \text { si } \widetilde{\mathrm{e}} \in\left[\mathbf{e}_{1}, \mathbf{e}_{2}\right] \text {, } \\
& \overline{\mathrm{e}}=\mathrm{e}_{1} \quad \text { si } \widetilde{\mathrm{e}}<\mathbf{e}_{1}, \\
& \overline{\mathrm{e}}=\mathbf{e}_{2} \quad \text { si } \tilde{\mathrm{e}}>\mathbf{e}_{2} \text {. }
\end{aligned}
$$

En conséquence, pour la population globale d'étudiants, la proportion d'individus qui resteront dans le pays d'origine est $\frac{\mathrm{N}_{0}}{\mathrm{~N}}=\frac{\overline{\mathrm{e}}-\mathrm{e}_{1}}{\mathrm{e}_{2}-\mathrm{e}_{1}}-$, alors que la 
proportion d' individus qui partiront étudier à l'étranger est le complément, soit $\frac{\mathrm{N}-\mathrm{N}_{0}}{\mathrm{~N}}=\frac{\mathrm{e}_{2}-\overline{\mathrm{e}}}{\mathrm{e}_{2}-\mathrm{e}_{1}}$.

\section{2. Production et bien-être dans le pays d'origine}

La production dans le pays d'origine, en fait la valeur ajoutée, est supposée être proportionnelle à la productivité. C'est ainsi que, si aucun individu n'allait étudier à l'étranger, elle serait $Y_{0}=e_{0} N$. De la même façon la valeur ajoutée engendrée par les diplômés locaux s'exprime par $\mathrm{e}_{0} \mathrm{~N}_{0}$. Comme la productivité des diplômés à l'étranger est supposée répartie uniformément, l'accroissement marginal de productivité correspondant à une proportion marginale $\mathrm{dn}$, de la population totale, $\mathrm{N}$, est tel que $\frac{\mathrm{dn}}{\mathrm{N}}=\frac{\mathrm{de}}{\mathrm{e}_{2}-\mathrm{e}_{1}}$. Cependant, il n'y a qu'une fraction (1-p(e)) de la population partie étudier à l'étranger qui va rentrer travailler dans le pays d'origine. Le nombre total des rentrants peut donc être exprimé par $\mathrm{N}_{1}=$ $\frac{\mathrm{N}}{\mathrm{e}_{2}-\mathrm{e}_{1}} \int_{\frac{\mathrm{e}}{2}}^{\mathrm{c}_{2}}[1-\mathrm{p}(\mathrm{e})] \mathrm{de}$. Leur contribution à la valeur ajoutée nationale est donc $\mathbf{Y}_{1}=\int_{\mathrm{N}_{0}}^{\mathrm{N}} \mathrm{e}[1-\mathrm{p}(\mathrm{e})] \mathrm{dn}=\frac{\mathrm{N}}{\mathrm{e}_{2}-\mathrm{e}_{1}} \int_{\mathrm{e}}^{\mathrm{e}} \mathrm{e}[1-\mathrm{p}(\mathrm{e})] \mathrm{de}$.

De la même façon, le nombre d'individus éduqués à l'étranger et qui y restent est $N_{1} *=\frac{N}{e_{2}-e_{1}} \int_{\frac{e}{2}}^{e_{2}^{2}} p(e) d e$. On rappelle également que le nombre d'individus qui ne partent pas à l'étranger s'exprime par $\mathbf{N}_{0}=\mathbf{N}-\left(\mathbf{N}_{1}+\mathbf{N}_{1}^{*}\right)=\mathrm{N} \frac{\overline{\mathrm{e}}-\mathrm{e}_{1}}{\mathrm{e}_{2}-\mathrm{e}_{1}}$.

Sur ces expressions, il est facile de voir que

a) $\quad \mathbf{N}_{0}$ est une fonction croissante du seuil de productivité, $\bar{e}$, alors que $N_{1}$ et $\mathrm{N}_{1}$ * en sont des fonctions décroissantes

b) $\quad N_{1}$ et $N_{1}^{*}$ croissent avec $e_{1}$ pour $e_{2}$ donné, ou avec $e_{1}$ et $e_{2}$ pour une valeur donnée de $e_{2}-e_{1}$. $N_{1}$ *croît aussi avec $e_{2}$ pour $e_{1}$ donné mais en revanche, $\mathrm{N}_{1}$ passe par un maximum pour une certaine valeur de $e_{2}$. Audelà de cette valeur limite, un accroissement du niveau de productivité atteint par les meilleurs étudiants à l'étranger va accentuer la fuite.

En prenant comme situation de référence l'autarcie, dans laquelle les individus n'ont pas la possibilité d'aller étudier à l'étranger et donc d'y trouver du travail, on peut expliciter la variation de bien-être engendrée par l'ouverture de cette possibilité. Nous exprimons cette variation comme la valeur ajoutée supplémentaire que permet le retour des diplômés moins 
le coût supplémentaire entraîné pour la collectivité par les départs à l'étranger.

On suppose ici que le coût des études à l'étranger est assumé par des agents du pays d'origine (les familles par exemple) quelle que soit la localisation future des individus. Ce coût concerne donc l'ensemble des étudiants qui partent à l'étranger ${ }^{8}$.

Cette variation de bien-être, $\Delta \mathrm{W}$, peut donc s'écrire

$\Delta \mathrm{W}=\mathbf{Y}_{1}-\mathbf{e}_{0}\left(\mathbf{N}-\mathbf{N}_{0}\right)-\delta\left(\mathbf{N}-\mathbf{N}_{0}\right)$

où $\delta=I^{*}-\mathbf{c}$, $\mathbf{c}$ étant le coût unitaire pour la collectivité des études locales. Par la suite, on suppose pour simplifier que les étudiants locaux paient ce qu'ils coûtent à la collectivité, donc que $I_{0}=c$. On peut alors écrire $\delta=\mathbf{i}$, d'où $\Delta W=Y_{1}-\left(e_{0}+i\right)\left(N-N_{0}\right)$.

On pose $C=e_{0}+\delta=e_{0}+i$. C reflète le coût d'opportunité social de l'éducation étrangère constitué par deux éléments : la perte de production et le différentiel de coût entre études à l'étranger et études locales.

De façon plus explicite, on peut écrire

$$
\text { (5.) } \Delta \mathbf{W}=\frac{\mathrm{N}}{\mathrm{e}_{2}-\mathrm{e}_{1}} \int_{\bar{e}}^{e_{2}}\langle\mathrm{e}[1-\mathrm{p}(\mathrm{e})]-\mathrm{C}\rangle \mathrm{de}
$$

Ceci est équivalent à

(6.) $\Delta \mathbf{W}=\frac{\mathrm{N}}{\mathrm{e}_{2}-\mathrm{e}_{1}} \int_{\bar{e}}^{e_{2}}\left[\mathrm{e} \frac{\mathrm{E}_{2}-\mathrm{e}}{\mathrm{E}_{2}-\mathrm{E}_{1}}-\mathrm{C}\right] \mathrm{de}=\frac{\mathrm{N}}{\mathrm{e}_{2}-\mathrm{e}_{1}} \int_{e}^{e_{2}} \varphi(\mathrm{e}) \mathrm{de}=\mathrm{N} \frac{\Phi\left(\mathrm{e}_{2}\right)-\Phi(\overline{\mathrm{e}})}{\mathrm{e}_{2}-\mathrm{e}_{1}}$

où $\varphi(e)=e \frac{E_{2}-e}{E_{2}-E_{1}}-C$, et $\Phi$ est une primitive de $\varphi$ :

$\Phi(e)=-\frac{e^{3}}{3\left(E_{2}-E_{1}\right)}+\frac{E_{2} e^{2}}{2\left(E_{2}-E_{1}\right)}-C e$.

L'équation (6.) montre que la variation de bien-être est fonction de tous les paramètres du modèle. Ainsi, elle dépend de :

_ $\mathrm{e}_{0}$ : la productivité des travailleurs éduqués à l'étranger;

- $e_{1}$ et $e_{2}$ : Les limites de l'intervalle dans lequel se trouvent les valeurs de la productivité pour les travailleurs éduqués à l'étranger ; $E_{1}$ et $E_{2}$ : paramètres affectant les conditions d'accès au marché étranger des travailleurs qualifiés, qui se répercutent sur la probabilité d'y trouver du travail ;

\footnotetext{
${ }^{8}$ On pourrait envisager une hypothèse alternative selon laquelle ce coût est assumé par l'individu lui-même et sera imputé sur son salaire futur. Dans ce cas, le coût à prendre en compte dans l'expression de la variation de bien-être ne concernerait que les étudiants qui rentrent dans le pays d'origine. Il est important de noter qu'on fait abstraction ici des éventuelles contraintes financières intertemporelles qui peuvent jouer un rôle important pour dissuader des individus d'investir dans le capital humain. De telles contraintes sont très liées à des problèmes d'inégalité de revenus et ressources, ainsi que de pauvreté, qui sont endémiques dans beaucoup de pays en voie de développement.
} 
_ $\overline{\mathrm{e}}$ : le seuil de productivité à partir duquel un individu décidera d'aller étudier à l'étranger; celui-ci dépend lui-même des salaires pratiqués sur les deux marchés du travail;

_ i : le différentiel de coût pour l'étudiant entre les études à l'étranger et les étude locales.

La fonction $\Phi$ dans l'équation (6.) est du troisième degré en e. Comme le montre la Figure 3, sa forme générale peut montrer tout d'abord un minimum, pour $\mathrm{e}=\hat{\mathrm{e}}_{1}$, puis un maximum pour $\mathrm{e}=\hat{\mathrm{e}}_{2}$. Les valeurs de $\hat{\mathrm{e}}_{1}$ et $\hat{e}_{2}$ sont respectivement données par $\hat{e}_{1}=\frac{1}{2}\left[E_{2}-\sqrt{E_{2}^{2}-4\left(E_{2}-E_{1}\right) C}\right]$ et $\hat{\mathrm{e}}_{2}=\frac{1}{2}\left[\mathrm{E}_{2}+\sqrt{\mathrm{E}_{2}^{2}-4\left(\mathrm{E}_{2}-\mathrm{E}_{1}\right) \mathrm{C}}\right]$. Naturellement, ces extrema existent seulement si $E_{2}^{2}-4\left(E_{2}-E_{1}\right) C>0$, soit encore

$$
\text { (7.) } \mathrm{C}<\frac{\mathrm{E}_{2}^{2}}{4\left(\mathrm{E}_{2}-\mathrm{E}_{1}\right)} \text {. }
$$

Si le coût social de l'éducation à l'étranger, $C$, est trop élevé, $\Phi$ est toujours décroissant avec e, et par conséquent $\Delta W$ est toujours négatif. II n'est donc pas souhaitable d'envoyer des étudiants à l'étranger. La valeur $\hat{e}_{1}$ pour laquelle $\Phi$ passe par un minimum n'est pertinente que si elle est plus grande que $E_{1}$. Les calculs montrent que la condition pour cela est simplement

$$
\text { (8.) } \quad \mathrm{E}_{1}<\mathrm{C} \text {. }
$$

Nous supposerons par la suite que les conditions (7.) et (8.) sont toujours satisfaites.

On peut remarquer également que la courbe admet un point d'inflexion pour $e=E_{2} / 2$, qui correspond au maximum de la fonction $\varphi$. Ce point est intéressant car c'est pour cette valeur du seuil $\bar{e}$ que l'augmentation marginale de bien-être associée à une diminution marginale de è est la plus forte. 


\section{Figure 3}

Forme de la fonction $\Phi$ représentant le bien-être

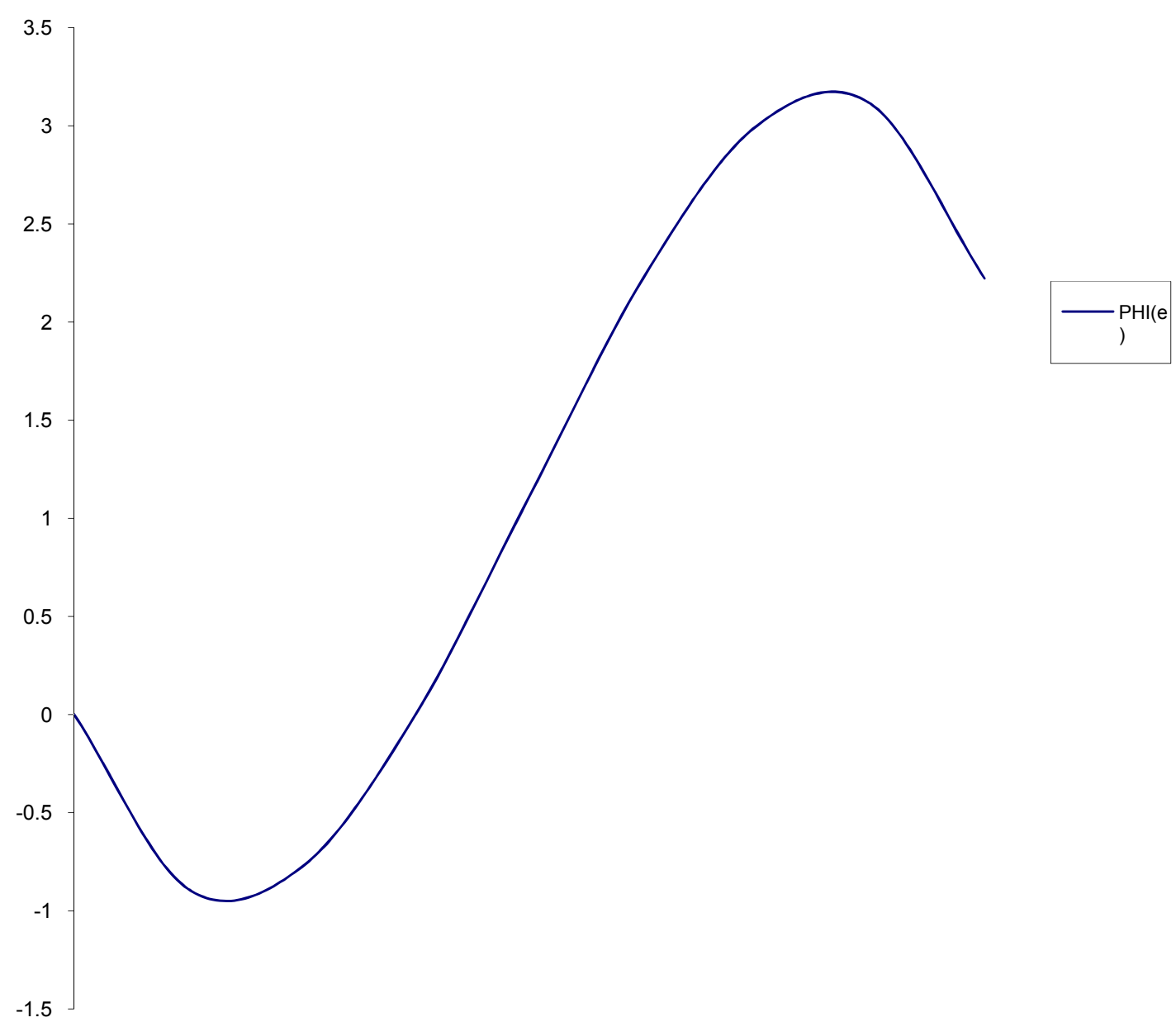

$\underline{\text { II.3 La relation entre le seuil de productivité et le bien-être }}$

On recherche ici quelle est l'influence du seuil $\overline{\mathrm{e}}$ sur la variation de bienêtre engendrée par le départ des étudiants à l'étranger.

D'après (6.), la variation de bien-être $\Delta W$ sera positive si $\Phi(\bar{e})<$ $\Phi\left(e_{2}\right)$. L'examen de la Figure 3 montre aisément que $\Delta W$ sera maximale pour $\overline{\mathrm{e}}=\hat{\mathbf{e}}_{1}$. Donc si initialement $\overline{\mathrm{e}}<\hat{\mathbf{e}}_{1}$, un accroissement marginal de $\overline{\mathrm{e}}$ est favorable au bien-être. En revanche, si $\overline{\mathrm{e}}>\hat{\mathbf{e}}_{1}$, un accroissement de $\overline{\mathrm{e}}$ réduit le nombre des départs et sera défavorable pour la collectivité. $\mathrm{Si} \overline{\mathrm{e}}>$ $\hat{\mathbf{e}}_{2}, \Delta \mathrm{W}$ sera toujours négative. De façon générale, on peut énoncer : 


\section{Proposition 1}

Pour des valeurs intermédiaires de $\overline{\mathrm{e}}$ et $\mathrm{e}_{2}$, à savoir appartenant à l'intervalle $\left[\hat{e}_{1}, \hat{e}_{2}\right]$, la possibilité d'aller étudier à l'étranger augmente le bien-être. Ainsi, l'effet positif du retour sur le bien-être domine l'influence négative de la fuite des cerveaux.

En revanche, il y a trois cas dans lesquels les études à l'étranger engendrent une perte de bien-être :

a) quand $\overline{\mathrm{e}}$ et $\mathrm{e}_{2}$ sont tous les deux très bas, le rendement social de l'éducation à l'étranger en termes d'accroissement de la productivité est faible et ne compense pas son coût même si beaucoup d'individus partent étudier puis reviennent travailler dans leur pays d'origine;

b) quand $\overline{\mathrm{e}}$ et $\mathrm{e}_{2}$ sont tous les deux très élevés, peu d'individus partent pour étudier mais la plupart de ceux-ci vont trouver du travail à l'étranger, d'où une influence dominante de la fuite ;

c) quandēest bas et $e_{2}$ est élevé, les effets mentionnés sous a) et b) peuvent se combiner de telle sorte que beaucoup d'individus partent ( d'où un coût d'opportunité élevé ), mais seuls ceux dont la productivité est la plus faible reviennent travailler dans le pays d'origine.

En résumé, l’impact des migrations suscitées par les études supérieures à l'étranger sur le bien-être est fondamentalement ambigu.

II.4 Implications pour le bien-être de changements des paramètres de productivité

Il est facile de voir qu'un accroissement de $e_{0}$, l'efficacité des diplômés locaux, et de $e_{1}$, la limite inférieure de la productivité des étudiants ayant accompli leurs études à l'étranger, ont respectivement une influence négative et positive sur le bien-être. Une hausse de $e_{0}$ accroît le coût d'opportunité des études à l'étranger, alors qu'une hausse de $e_{1}$ ( à $e_{2}$ constant) accroît le rendement de l'éducation à l'étranger.

Les effets d'une variation de $e_{2}$ sont plus complexes à analyser et nécessitent l'examen de la dérivée de $\Delta \mathrm{W}$,donnée par la formule

$$
\left(\mathbf{e}_{2}-\mathbf{e}_{1}\right)^{2} \frac{1}{N} \frac{\mathrm{d} \Delta \mathrm{W}}{\mathrm{de}_{2}}=\Phi(\overline{\mathrm{e}})-\left[\Phi\left(\mathbf{e}_{2}\right)-\left(\mathbf{e}_{2}-\mathbf{e}_{1}\right) \varphi\left(\mathbf{e}_{2}\right)\right] \text {. }
$$

On voit que si $\overline{\mathrm{e}}<\hat{\mathbf{e}}_{2}$, de telle sorte que $\Delta W$ puisse être positive,alors l'expression ci-dessus est positive pour $\mathrm{e}_{2}=\overline{\mathrm{e}}$ et négative pour $\mathbf{e}_{2}=\hat{\mathbf{e}}_{2}$. Le bien-être admet donc un maximum pour une certaine valeur de $e_{2}$ (également inférieure à ê $\hat{e}_{2}$ ). On peut donc énoncer : 


\section{$\underline{\text { Proposition } 2}$}

La variation de bien-être dans le pays domestique, $\Delta W$, est une fonction croissante de la valeur de $e_{2}$, le niveau maximum de productivité que peuvent atteindre les meilleurs étudiants à l'étranger, tant que $e_{2}$ reste au-dessous d'une certaine valeur critique, au-delà de laquelle $\Delta W$ décroît.

Ainsi, un accroissement trop important du capital humain, ou, si l'on veut, trop d'élitisme dans les institutions académiques étrangères entraîne une trop grande fuite des cerveaux et, de ce fait, est nuisible au bien-être du pays domestique.

Finalement, si $e_{1}$ et $e_{2}$ augmentent tous deux en conservant un écart constant, le maximum de $\Delta \mathrm{W}$ correspond à $\mathrm{e}_{2}=\hat{\mathbf{e}}_{2}$. L'efficacité optimale est donc plus élevée que dans le cas précédent, mais là encore tout dépassement de ce niveau entraînerait une réduction du bien-être.

Section IV: Influence sur le bien-être de l'attribution de bourses à des étudiants désireux de partir à l'étranger

\section{IV.1 Principe}

On a vu dans ce qui précède que le fait d'ouvrir la possibilité à des individus d'aller étudier à l'étranger pouvait sous certaines conditions avoir des conséquences positives pour le bien-être grâce à une dominance du phénomène du retour des diplômés. Il est donc logique dans cette situation d'essayer d'augmenter ce gain en amplifiant les départs, et ceci en abaissant le coût des études à l'étranger pour un étudiant par l'intermédiaire d'un système de bourses.

On suppose ici que l'administration est capable d'identifier la capacité individuelle et donc la productivité future de chaque étudiant. Elle est donc amenée à proposer des bourses aux étudiants qui se situent en - dessous du seuil de productivité $\overline{\mathrm{e}}$ au delà duquel un étudiant non boursier décidera de partir à l'étranger. L'effet de la bourse sera de réduire le coût $I^{*}$ du départ à l'étranger et donc d'abaisser le seuil de productivité à partir duquel le départ devient rentable pour un étudiant. Intuitivement, on est tenté d'accorder une bourse aux meilleurs des candidats, c'est-à-dire ceux dont la productivité est la plus proche du seuil $\bar{e}$. Il faut observer cependant que ces étudiants sont aussi ceux pour lesquels la probabilité d'accéder au marché du travail étranger est la plus forte, amplifiant ainsi la fuite des cerveaux. On peut donc se demander s'il ne serait pas préférable de subventionner des étudiants moins brillants, mais dont la probabilité de 
retour est plus élevée. On va donc supposer a priori que les bourses seront attribuées à des candidats dont la productivité est comprise entre deux limites $\overline{\mathrm{e}}_{\mathrm{s}}$ et $\varepsilon$, avec $\varepsilon \leq \overline{\mathrm{e}}$. L'étendue des bourses distribuées dépend naturellement de la contrainte budgétaire qui s'impose à l'administration que nous introduirons ici par l'intermédiaire d'un coût d'opportunité des fonds publics $\lambda$.

Soit $S$ le montant d'une bourse. Le coût supplémentaire pour l'étudiant boursier qui décide de partir à l'étranger est maintenant $\mathbf{i}-\mathrm{S}$. Pour ce type d'étudiant, il existe maintenant une nouvelle probabilité limite qui s'exprime par : $\overline{\mathrm{p}}_{\mathrm{s}}=\frac{\mathrm{i}-\mathrm{S}-\left(\mathrm{w}_{1}-\mathrm{w}_{0}\right)}{\mathrm{w}^{*}-\mathrm{w}_{1}}=\overline{\mathrm{p}}-\frac{\mathrm{S}}{\mathrm{w}^{*}-\mathrm{w}_{1}}$

Par ailleurs, $\overline{\mathrm{e}_{\mathrm{s}}}=\left(\mathrm{E}_{2}-\mathbf{E}_{1}\right) \overline{\mathrm{p}}_{\mathrm{s}}+\mathrm{E}_{1}$ et $\overline{\mathrm{e}}-\overline{\mathrm{e}_{\mathrm{s}}}=\left(\mathrm{E}_{2}-\mathbf{E}_{1}\right)\left(\overline{\mathrm{p}}-\overline{\mathrm{p}}_{\mathrm{s}}\right)$. En posant $\Delta=E_{2}-E_{1}$ et $\delta^{*}=w^{*}-w_{1}$, on en déduit la relation entre $S$ et $\mathrm{e}_{\mathrm{s}}$ : $\mathbf{S}=\frac{\delta^{*}}{\Delta}\left(\overline{\mathrm{e}}-\overline{\mathrm{e}_{\mathrm{s}}}\right)$.

L'attribution de bourses entraîne une variation de bien-être supplémentaire ( qui s'ajoute à la variation $\Delta W$ engendrée en l'absence de bourses). Celleci tient compte de deux considérations : d'une part, plus d'individus vont partir dont une partie va ensuite rentrer dans le pays d'origine, d'où un nouvel accroissement de la valeur ajoutée. Mais s'il existe un coût d'opportunité des fonds publics, $\lambda$, cela veut dire que si un étudiant paie maintenant $I^{*}-S$ en partant à l'étranger, il va coûter à l'Etat $(1+\lambda) S$. Le coût social supplémentaire net par étudiant est donc $\lambda S$.

La variation correspondante de bien-être s'exprime donc par

$\Delta \mathrm{W}_{\mathrm{s}}=\frac{\mathrm{N}}{\mathrm{e}_{2}-\mathrm{e}_{1}} \int_{\bar{e}_{\mathrm{s}}}^{\varepsilon}[\varphi(\mathbf{e})-\lambda \mathrm{S}] \mathbf{d e}$

\section{IV.2 Détermination des bénéficiaires}

$A \overline{\mathrm{e}_{\mathrm{s}}}$ donné, si on cherche à maximiser $\Delta W_{\mathrm{s}}$ par rapport à $\varepsilon$, on voit que de deux choses l'une :

_ soit $\varphi(\overline{\mathrm{e}}) \geq \lambda$ S et alors $\varepsilon^{*}=\overline{\mathrm{e}}$,

_ soit $\varphi(\mathrm{e})<\lambda \mathrm{S}$ et alors $\varepsilon^{*}$ est tel que $\varphi\left(\varepsilon^{*}\right)=\lambda \mathrm{S}$.

$\bar{O}$ peut déjà remarquer que si $\overline{\mathrm{e}}>\hat{\mathrm{e}}_{2}, \varphi(\overline{\mathrm{e}})<0$, on est donc dans le deuxième cas et $\varepsilon$ ne sera certainement pas égal à $\overline{\mathrm{e}}$. Par ailleurs si $\overline{\mathrm{e}}<\mathbf{E}_{2} /$ $2, \varepsilon *<\overline{\mathrm{e}}$ implique $\varphi\left(\varepsilon^{*}\right)<\varphi(\overline{\mathrm{e}})$, ce qui est incompatible avec le deuxième cas. On a donc alors $\varepsilon^{*}=\overline{\mathrm{e}}$.

On s'intéresse maintenant au cas où $E_{2} / 2<\overline{\mathrm{e}}<\hat{\mathbf{e}}_{2}$. 
Si $\overline{\mathrm{e}_{\mathrm{s}}}$ est tel que $\varphi(\overline{\mathrm{e}})<\lambda \mathrm{S}$, les valeurs optimales, $\overline{\mathrm{e}_{\mathrm{s}}} *$ et $\varepsilon^{*}$, de $\overline{\mathrm{e}_{\mathrm{s}}}$ et $\varepsilon$ sont données par les équations (9.) et (10.) :

(9.) $\varphi\left(\varepsilon^{*}\right)=\lambda S$

(10.) $\frac{\mathrm{d} \Delta W_{\mathrm{s}}}{\mathrm{de}_{\mathrm{s}}}=\frac{\mathrm{N}}{\mathrm{e}_{2}-\mathrm{e}_{1}}\left[\varphi\left(\overline{\mathrm{e}_{\mathrm{s}}} *\right)+\lambda \mathrm{s}-\lambda\left(\varepsilon *-\overline{\mathrm{e}_{\mathrm{s}}} *\right) \frac{\mathrm{dS}}{\mathrm{de}_{\mathrm{s}}}\right]=\mathbf{0}$

$\operatorname{avec} \frac{\mathrm{dS}}{\mathrm{de}_{\mathrm{s}}}=-\frac{\delta *}{\Delta}$

En éliminant $S$ entre les deux équations, il vient

(11.) $\frac{\varphi\left(\varepsilon^{*}\right)-\varphi\left(\overline{\mathrm{e}_{\mathrm{s}}^{*}}\right)}{\varepsilon^{*}-\overline{\mathrm{e}_{\mathrm{s}}^{*}}}=-\lambda \frac{\delta^{*}}{\Delta}$

On voit que ceci implique $\varphi\left(\varepsilon^{*}\right)<\varphi\left(\overline{\mathrm{e}_{\mathrm{s}}} *\right)$.

Compte tenu de l'expression de la fonction $\varphi$, on obtient finalement la condition

(12.) $\varepsilon^{*}+\overline{\mathrm{e}_{\mathrm{s}}} *=\mathbf{E}_{2}+\lambda \delta^{*}$,

condition qui implique $\varepsilon^{*}>E_{2} / 2$.

On peut envisager le cas limite où les équations (9.) et (12.) admettent pour solution $\varepsilon^{*}=\overline{\mathrm{e}}:$ Elles donnent alors :

$$
\begin{aligned}
& \text { (13.) } \varphi(\overline{\mathrm{e}})=\lambda \mathrm{S}=\frac{\lambda \delta^{*}}{\Delta}\left(\overline{\mathrm{e}}-\overline{\mathrm{e}_{\mathrm{s}}} *\right) \\
& \text { (14.) } \overline{\mathrm{e}}+\overline{\mathrm{e}_{\mathrm{s}}} *=\mathbf{E}_{2}+\lambda \delta^{*}
\end{aligned}
$$

En éliminant $\overline{\mathrm{e}_{\mathrm{s}}} *$, il vient

$$
\text { (15.) } \quad 2 \overline{\mathrm{e}}-\mathbf{E}_{2}=\lambda \delta *+\frac{\Delta}{\lambda \delta^{*}} \varphi(\overline{\mathrm{e}})=\mathbf{f}(\lambda \delta *)
$$

La fonction f passe par un minimum pour une valeur $\hat{\lambda}$ de $\lambda$ telle que $\hat{\lambda} \delta^{*}=\sqrt{\Delta \varphi(\overline{\mathrm{e}})}$

Cette équation montre que pour è donné, il peut exister deux valeurs de $\lambda$ pour lesquelles cette condition est satisfaite, $\lambda_{1}$ et $\lambda_{2}$ telles que $\lambda_{1} \leq \hat{\lambda} \leq \lambda_{2}$. Plus précisément, cela sera le cas si $\overline{\mathrm{e}}-\mathbf{E}_{2} / 2 \geq \sqrt{\Delta \varphi(\overline{\mathrm{e}})}$, ce qui implique que e soit supérieur ou égal à une certaine valeur $\mathrm{e}_{\min }$ telle que

$\mathbf{E}_{\mathbf{2}} / \mathbf{2} \leq \mathbf{e}_{\min } \leq \hat{\mathbf{e}}_{\mathbf{2}}$

On montre en annexe que si $\overline{\mathrm{e}} \geq \mathbf{e}_{\min }$ et si $\left.\lambda \in\right] \lambda_{1}, \lambda_{2}[$, la valeur optimale de $\varepsilon$ est strictement inférieure à $\overline{\mathrm{e}}$.

On peut donc énoncer la

\section{Proposition 3}


Pour un seuil de productivité è donné,

a) si $\overline{\mathrm{e}}<\mathbf{e}_{\min }$, tous les étudiants dont la productivité est comprise entre une valeur minimale dépendant du coût d'opportunité des fonds publics et le seuil doivent recevoir une bourse; il en est de même si $\mathbf{e}_{\min }<\overline{\mathrm{e}}<\hat{\mathbf{e}}_{2}$ et si le coût d'opportunité n'appartient pas à un certain intervalle $\left[\lambda_{1}, \lambda_{2}\right]$.

b) En revanche, si $\mathbf{e}_{\text {min }}<\overline{\mathrm{e}}<\hat{\mathbf{e}}_{2}$, et si le coût d'opportunité des fonds publics est compris entre $\lambda_{1}$ et $\lambda_{2}$, il ne faut pas donner de bourses aux étudiants dont la productivité serait inférieure à $\bar{e}$, mais proche de celui-ci. Il en est de même si $\overline{\mathrm{e}}>\hat{\mathbf{e}}_{2}$, et ceci quelle que soit la valeur du coût d'opportunité.

Dans ce cas b), La population totale se partage entre quatre catégories d'individus par ordre de capacités croissantes :

Ceux dont les capacités sont trop faibles pour qu'ils puissent rentabiliser leur départ à l'étranger, même s'ils recevaient une bourse ( $e_{k}$ plus petit que $\overline{\mathrm{e}_{\mathrm{s}}} *$ ) ; ceux qui partiront grâce à la bourse ( $\mathrm{e}_{\mathrm{k}}$ compris entre $\overline{\mathrm{e}_{\mathrm{s}}} *$ et $\left.\varepsilon^{*}\right)$; ceux qui ont une capacité supérieure à celles des précédents, mais qui ne partiront pas faute de bourse ( $e_{\mathrm{k}}$ compris entre $\varepsilon *$ et $\overline{\mathrm{e}}$ ); enfin ceux qui partiront sans bourse ( $e_{k}$ plus grand que $\left.\bar{e}\right)$.

Ce dernier résultat semble assez intuitif lorsque $\bar{e}>\hat{e}_{2}$ puisqu'on est alors dans une situation où, au voisinage de $\bar{e}$, le rendement marginal d'une diminution du seuil est négatif. Il est plus paradoxal lorsque $\overline{\mathrm{e}}<\hat{\mathbf{e}}_{2}$. Pour le comprendre, on peut a contrario analyser les situations dans lesquelles $\varepsilon^{*}$ se confond avec $\overline{\mathrm{e}}$, c'est-à-dire à la fois pour des valeurs faibles et fortes du coût d'opportunité :

Si $\lambda$ est faible, la contrainte budgétaire est légère et on peut doter le maximum d'étudiants. On peut prendre $\overline{\mathrm{e}}_{\mathrm{s}}$ proche de $\hat{\mathrm{e}}_{1}$, mais plus $\overline{\mathrm{e}}_{\mathrm{s}}$ se rapproche de $\hat{e}_{1}$, plus la rentabilité de la bourse marginale est faible ; à partir d'un certain moment, il vaut donc mieux augmenter $\varepsilon$.

Si $\lambda$ est élevé, la contrainte financière est forte. Le montant de la bourse ainsi que le nombre de boursiers seront faibles. Partant d'une situation donnée, si l'on veut augmenter le nombre de bourses pour accroître le bienêtre, on peut procéder de deux façons, soit en augmentant $\varepsilon$, soit en diminuant $\overline{\mathrm{e}}_{\mathrm{s}}$. Mais, dans cette deuxième éventualité, on augmente aussi le montant de la bourse, et si le coût d'opportunité est élevé, cela va avoir des conséquences fâcheuses sur le bien-être. Il vaut donc mieux augmenter $\varepsilon$ et lui laisser atteindre $\overline{\mathrm{e}}$. 
On peut alors comprendre que, pour des valeurs intermédiaires de $\lambda$, on cherchera plutôt à profiter du fait que la rentabilité de la bourse marginale, matérialisée par la valeur de la fonction $\varphi$, est plus élevée au voisinage de $\overline{\mathrm{e}}_{\mathrm{s}}$ qu'au voisinage d' $\varepsilon$; il vaut donc mieux diminuer $\overline{\mathrm{e}}_{\mathrm{s}}$ qu'augmenter $\varepsilon$.

On voit donc que la politique optimale d'attribution des bourses ne favorise pas toujours les meilleurs étudiants. Ce paradoxe fait penser aux effets de seuil qui apparaissent chaque fois qu'une subvention est soumise à certaines conditions pour être attribuée à des individus, notamment une contrainte de ressources maximales : le candidat qui se situera juste en dessous du seuil sera alors avantagé par rapport à celui qui dépasse le seuil, même de très peu. La conséquence d'une telle situation est que les individus seront tentés de dissimuler leurs ressources pour pouvoir avoir droit à la subvention. Le même problème se pose ici : un candidat boursier sera incité à se comporter de telle sorte que l'administration sous-estime ses capacités, et il n'est pas facile d'imaginer des mesures réalistes qui pourraient l'en empêcher. Donc, l'implémentation d'une telle politique de sélection n'est pas cohérente («Incentive compatible») par rapport aux incitations à l'excellence académique.

\section{Section V: Conclusion}

Un aspect distinctif de la modélisation proposée dans cet article est l'analyse de l'impact du choix d'investir en capital humain à l'étranger sur le bien-être d'un pays d'origine. Contrairement à une grande partie de la littérature, la probabilité d'obtenir un emploi à l'étranger est endogénéisée. Elle dépend précisément des capacités des individus et de leur choix de poursuivre leurs études à l'étranger. Un résultat central est qu'il est en général difficile d'apporter une réponse tranchée à la question de savoir si l'impact de la possibilité d'aller faire des études à l'étranger sur le bien-être du pays d'origine est positif ou négatif. Cela est du à la non-linéarité des effets opposés sur le bien-être engendrés par les phénomènes de «brain drain» et «brain gain». Un élément clef est que les individus les plus talentueux ont une plus forte probabilité de rester à l'étranger, tandis que le financement de leurs études impose un coût social supplémentaire au pays d'origine.

Néanmoins, lorsque la productivité limite qui détermine quels individus vont partir et la productivité maximale que peuvent atteindre les individus éduqués à l'étranger prennent toutes deux des valeurs moyennes par rapport aux bornes de leur intervalle de variation, l'effet net sur le bienêtre est positif car l'effet du retour domine celui de la fuite des cerveaux. En revanche il existe des valeurs extrêmes de ces paramètres de productivité pour lesquelles l'impact net sur le bien-être est négatif. C'est en particulier le cas lorsque le gain de productivité engendré par les études à l'étranger 
est trop élevé. Ceci peut paraître constituer un premier paradoxe, mais s'explique par la hausse de la probabilité que les étudiants soient par la suite absorbés par le marché étranger.

Un système de bourses peut accroître les effets positifs de l'ouverture sur l'étranger mais parfois seulement si la contrainte budgétaire n'est pas trop forte. On peut alors, si le seuil de productivité est assez élevé et sous certaines limites de la contrainte budgétaire, observer un second paradoxe qui découle du constat qu'il n'est pas toujours optimal d'attribuer des bourses aux candidats les plus méritants. Remarquons seulement que ce résultat pourrait être invalidé si on introduisait des «bourses conditionnelles ", imposant une obligation de retour aux boursiers?

Nous nous sommes intéressés ici à l'enseignement supérieur, en considérant comme déjà élaborés les choix publics dans le pays d'origine en matière d'éducation concernant les cycles précédents. Les dépenses correspondantes apparaissent ainsi comme des coûts irréversibles qui n'ont pas d'influence sur les décisions des agents publics et privés. Il serait possible d'étendre l'horizon temporel de l'analyse et d'endogénéiser ces choix en anticipant les conséquences qu'ils peuvent avoir sur le «brain drain » et le « brain gain ». Surtout dans les pays en voie de développement, il existe une contrainte budgétaire forte sur l'emploi des fonds publics. On peut donc se demander si, compte tenu du degré de risque de fuite, il est bien raisonnable de privilégier une utilisation élitiste des ressources budgétaires limitées pour favoriser la formation des plus capables. Peutêtre vaut-il mieux viser l'éducation primaire et secondaire pour l'ensemble de la population sachant que cette politique est moins affectée par le phénomène du « brain drain ».

Dans cet article, nous n'avons analysé que le bien-être dans le pays d'origine, sans considérer les implications pour le pays d'accueil. Une analyse plus générale pourrait considérer un jeu stratégique entre les deux pays. Dans un tel cadre, les instruments stratégiques pourraient être entre autres la politique des bourses et les politiques de prix de la scolarité dans les deux pays, avec éventuellement la possibilité pour le pays d'accueil de discriminer entre étudiants étrangers et locaux. En outre, les politiques d'immigration/visa peuvent modifier les seuils de productivité qui déterminent la probabilité d'accès au marché du travail étranger et ainsi affecter l'équilibre entre retour et fuite des cerveaux au bénéfice du pays d'accueil' ${ }^{10}$.

\footnotetext{
${ }^{9}$ A condition, naturellement,que cette obligation puisse effectivement être contrôlée de façon efficace, ce qui n'est pas forcément évident.

${ }^{10}$ De telles politiques reflètent une stratégie du type «beggar-thy-neighbour ».
} 


\section{Bibliographie}

Beine M., F. Docquier, et H. Rapoport (2001), "Brain Drain and economic growth : theory and evidence", Journal of Development Economics, Vol. 64, pp.275-289.

Beine M., F. Docquier, et H. Rapoport (2008), "Brain drain and human capital formation in developing countries: winners and losers", Economic Journal, Vol. 118, pp. 631-652 .

Bhagwati J. et K. Hamada (1974), "The brain drain, international integration of markets for professional and unemployment", Journal of Development Economics, 1, pp. 19-24.

Chiswick B. (1999), "Are Immigrants Favorably Self-Selected ? ", American Economic Review, Vol. 89, N², pp. 181-185.

Fan C. S. et O. Stark (2007), "International migration and "educated unemployment" ", Journal of Development Economics, Vol. 83, pp. 76-87.

Franck B. et R. F. Owen (2009), "International Human Capital Formation, Brain Drain and Brain Gain : A Conceptual Framework", document de travail, LEMNA, Université de Nantes.

Groizard J. et J. Llull (2006)," Brain drain or brain gain? New empirical evidence", communication au colloque de l' European Trade Study Group, Vienne, Septembre.

Katz E. et O. Stark (1987), "International Migration under Asymmetric Information", Economic Journal, 97(387), pp. 718-726.

Kwok V. et H. Leland (1982) "An Economic Model of the Brain Drain", American Economic Review,Vol.72, $\mathrm{N}^{\circ} 1$, pp. 91-100.

Lien D. et Y. Wang (2005), “ Brain drain or brain gain, A revisit ", Journal of Population Economics, Vol.18,pp. 153-163.

Mountford A. (1997) "Can a Brain Drain be Good for Growth in the Source Economy", Journal of Development Economics, 53(2), pp. 287- 303.

Niimi Y. et C. Özden (2007), "Brain Drain in Latin America and the Caribbean ", Working Paper, World Bank, Washington, March.

Özden C. et M. Schiff (2006) Editors, International Migration, Remittances and the Brain Drain, World Bank and Palgrave Macmillan, Washington.

Rosenzweig M.R. (2006), "Higher education and International Migration in Asia : Brain Circulation", manuscrit non publié, Yale University, Décembre 2006 ( préparé pour la Conférence Régionale sur l'Economie du Développement (RBCDE), Beijing, 16-17 Janvier 2007).

Schiff M. (2006), “ Brain Gain : Claims about its Size and Impact on Welfare and Growth Are Greatly Exaggerated", Ch. 5 de Özden C. 
et M. Schiff (2006) Editors, International Migration, Remittances and the Brain Drain, World Bank and Palgrave Macmillan, Washington.

Stark O. (2004), "Rethinking the Brain Drain", World Development, Vol. 32, No.1, pp. 15-22.

Stark O., C. Helmenstein, et A. Prskawetz (1997), "A Brain Gain with a Brain Drain", Economic Letters, Vol. 55, pp. 227-234.

Stark O., C. Helmenstein, et A. Prskawetz (1998), "Human Capital Depletion, Human Capital Formation, and Migration: a Blessing or a Curse?", Economic Letters, Vol.60, pp. 363-367.

Vidal J. (1998) "The Effect of Emigration on Human Capital Formation", Journal of Population Economics, Vol.11, pp. 589-600. 


\section{ANNEXE}

On rappelle tout d'abord que dans le cas où $\varphi(\overline{\mathrm{e}})<\lambda \mathrm{S}$, les valeurs optimales de $\overline{\mathrm{e}_{\mathrm{s}}}$ et $\varepsilon$ sont données, en posant $\lambda \delta^{*}=\lambda$, par les équations

(4.) $\varepsilon+\overline{\mathrm{e}_{\mathrm{s}}}=\mathbf{E}_{2}+\lambda \delta^{*}=\mathbf{E}_{2}+\lambda$,

(8.) $\varphi\left(\varepsilon^{*}\right)=\lambda \mathrm{S}=\frac{\lambda^{\prime}}{\Delta}\left(\overline{\mathrm{e}}-\overline{\mathrm{e}_{\mathrm{s}}}\right)$

Cette dernière peut également s'écrire

(9.) $\overline{\mathrm{e}}-\overline{\mathrm{e}_{\mathrm{s}}}=\frac{\Delta}{\lambda^{\prime}} \varphi\left(\varepsilon^{*}\right)$

On suppose que l'on se trouve initialement dans une situation où $\varepsilon^{*}=\overline{\mathrm{e}}$ et on considère à partir de là une variation marginale de $\lambda, d \lambda$.

En différentiant les équations (4.) et (9.), on trouve

$\mathbf{d} \varepsilon+\mathbf{d} \overline{\mathrm{e}_{\mathrm{s}}}=\mathbf{E}_{2}+\lambda \delta^{*}=\mathbf{d} \lambda$,

$\mathbf{d} \overline{\mathrm{e}_{\mathrm{s}}}=\frac{\Delta}{\lambda^{\prime 2}} \varphi(\overline{\mathrm{e}}) \mathbf{d} \lambda \lambda^{\prime}-\frac{\Delta}{\lambda^{\prime}} \varphi^{\prime}(\overline{\mathrm{e}}) \mathbf{d} \varepsilon$

et en éliminant $d \varepsilon$ entre ces équations, on obtient

$\left[1-\frac{\Delta}{\lambda^{\prime}} \varphi^{\prime}(\overline{\mathrm{e}})\right] \mathbf{d} \overline{\mathrm{e}_{\mathrm{s}}}=\left[\frac{\Delta}{\lambda^{\prime 2}} \varphi(\overline{\mathrm{e}})-\frac{\Delta}{\lambda^{\prime}} \varphi^{\prime}(\overline{\mathrm{e}})\right] \mathrm{d} \lambda \lambda^{\prime}$

Comme $\varphi^{\prime}(\bar{e}) \leq 0$, on constate que si $\lambda$ augmente, il en est de même de $\overline{e_{s}}$, ce qui est naturel : la population des boursiers se réduit au niveau des moins qualifiés.

On peut alors calculer $d \varepsilon$ :

$\mathbf{d} \varepsilon=\frac{\lambda^{\prime 2}-\Delta \varphi(\overline{\mathrm{e}})}{\lambda^{\prime}-\Delta \varphi^{\prime}(\overline{\mathrm{e}})} \frac{\mathrm{d}^{\prime} \lambda^{\prime}}{\lambda^{\prime}}$

En revenant à la définition de la fonction $\varphi$, on a $\Delta \varphi^{\prime}(\overline{\mathrm{e}})=\mathbf{E}_{2}-2 \overline{\mathrm{e}} \leq \mathbf{0}$. Le dénominateur de l'expression $\mathrm{de} d \varepsilon$ est donc positif.

Plaçons nous dans le cas où $\overline{\mathrm{e}}-\mathbf{E}_{2} / 2 \geq \sqrt{\Delta \varphi(\overline{\mathrm{e}})}$

Comme $\Delta \varphi(\overline{\mathrm{e}})=\overline{\mathrm{e}}\left(\mathbf{E}_{2}-\overline{\mathrm{e}}\right)-\Delta \mathbf{C}$, cette condition devient

$2\left(\overline{\mathrm{e}}-\mathrm{E}_{2} / 2\right)^{2} \geq \mathbf{E}_{2}{ }^{2} / 4-\Delta \mathrm{C}$, ou encore, en se souvenant de la définition de $\hat{\mathbf{e}}_{2}, 2\left(\mathrm{e}-\mathbf{E}_{2} / 2\right)^{2} \geq\left(\hat{e}_{2}-E_{2} / 2\right)^{2}$. Ceci implique $\bar{e}>\mathbf{e}_{\min }$, celui-ci étant défini par

$2\left(\mathrm{e}_{\min }-\mathbf{E}_{2} / 2\right)^{2} \geq\left(\hat{e}_{2}-E_{2} / 2\right)^{2}$ qui implique $\mathrm{E}_{2} / 2 \leq \mathrm{e}_{\min } \leq \hat{\mathrm{e}}_{2}$.

On sait que, dans ce cas, un optimum pour lequel $\varepsilon=\overline{\mathrm{e}}$ correspond à l'une de deux valeurs $\lambda_{1}$ et $\lambda_{2}$ du coût d'opportunité telles que $\lambda_{1} \leq \hat{\lambda} \leq \lambda_{2}$, avec $\hat{\lambda} \delta *=\sqrt{\Delta \varphi(\overline{\mathrm{e}})}$, donc $\delta * \lambda_{1} \leq \sqrt{\Delta \varphi(\overline{\mathrm{e}})} \leq \delta^{*} \lambda_{2}$. 
Si donc on choisit de retenir la plus petite valeur $\lambda_{1}$, on en déduit que $\lambda_{1}^{\prime 2}-\Delta \varphi(\overline{\mathrm{e}}) \leq 0$ et par conséquent le numérateur de l'expression de $\mathbf{d} \varepsilon$ est négatif. En définitive donc, si $\lambda$ augmente à partir de cette valeur initiale $\lambda_{1}$, $\varepsilon$ diminue. Comme on avait initialement $\varepsilon=\bar{e}$, la valeur optimale de $\varepsilon$ sera maintenant inférieure à $\mathrm{e}$.

Symétriquement, si l'on retient la plus grande valeur $\lambda_{2}$ de $\lambda$, le numérateur de $d \varepsilon$ est positif, et il en est de même $d e d \varepsilon / d \lambda$. Donc, si $\lambda$ diminue, de nouveau $\varepsilon$ diminue et on arrive au même résultat.

On peut donc finalement en conclure que, lorsque $\bar{e} \geq \mathbf{e}_{\min }$ et $\left.\lambda \in\right] \lambda_{1}, \lambda_{2}[$, la valeur optimale de $\varepsilon$ est strictement inférieure à $\bar{e}$. 